\title{
A novel particle tracking and break-up detection algorithm: application to the turbulent break-up of bubbles
}

\author{
Javier Rodríguez-Rıdríguez, Carlos Martínez-Bazán and \\ José Luis Montañes \\ Departamento de Ingeniería Térmica y de Fluidos, Universidad Carlos III de Madrid, \\ Avda. de la Universidad 30, 28911 Leganés (Madrid). Spain \\ Departamento de Motopropulsión y Termofluidodinámica, Universidad Politécnica de \\ Madrid, Pza. Cardenal Cisneros 3, 28040 Madrid, Spain \\ E-mail: carlosmb@ing.uc3m.es
}

\begin{abstract}
A new method has been developed to measure experimentally the break-up properties of bubbles. The technique is based on the application of a particle tracking velocimetry algorithm to high-speed video images not only to measure the velocity of the bubbles, but also to detect the break-up events. Thus the algorithm is able to associate every broken bubble with the daughter bubbles formed upon their corresponding break-up. Moreover, the lifetime, as well as the number and size of fragments resulting from the break-up process, can be measured for a large number of bubbles. Statistical processing of the information collected allows us to compute the break-up frequency and daughter size distribution of the bubbles as a function of the bubble size and the mean properties of the base flow. The method has been employed to study the break-up of a cloud of bubbles injected at the central axis of a turbulent water jet. Experimental results for the break-up frequency and daughter bubble size distribution are also presented to illustrate the performance of the technique.
\end{abstract}

Keywords: particle velocity, turbulent dissipation, bubble break-up, break frequency, particle tracking, high speed video, hot wire anemometry, image processing, computer vision, spray systems, atomization, multiphase flows

\section{Introduction}

The atomization of a gas cavity immersed in a turbulent flow is a phenomenon with a wide range of applications in both industrial as well as natural processes. It is well known that the bubble size distribution plays a key role in mass transfer processes such as those occurring in chemical reactors when reactants can be found in both gaseous and liquid phases. In addition, the exchange of certain gaseous species between the ocean and the atmosphere, e.g. $\mathrm{CO}_{2}$, is believed to be controlled by mass transfer processes occuring in the

\footnotetext{
3 Author to whom any correspondence should be addressed.
}

air bubbles produced by breaking waves. Reliable models of the turbulent break-up of gaseous cavities, i.e. bubbles, are crucial to describe the above-mentioned processes, thus allowing scientists and engineers to make accurate numerical predictions of practical interest.

The first work on the turbulent break-up of particles (droplets or bubbles) was due to Kolmogorov [9]. In a classical paper, Kolmogorov claimed that when the size of the particles is larger than the viscous scale of the turbulence, the only parameter governing the break-up process is the turbulent Weber number, defined as the ratio between the deforming stresses due to turbulent pressure fluctuations and the confining 
stresses due to surface tension

$$
W e_{\mathrm{t}}=\frac{\rho \overline{\Delta u^{2}}(D) D}{12 \sigma}
$$

where $\rho$ is the density of the continuous phase, $\overline{\Delta u^{2}}(D)$ is the squared difference in velocity between two points situated a distance apart of the order of the size of the particle, $D$, and $\sigma$ is the gas-liquid surface tension coefficient. Kolmogorov proposed the existence of a critical Weber number which determines whether a drop immersed in an isotropic flow field is stable or, on the contrary, breaks up in a given time.

When the particle's size, $D$, falls within the inertial subrange of the turbulent spectrum, the definition of the Weber number given by equation (1) may be rewritten as

$$
W e_{\mathrm{t}}=\frac{\beta \rho \varepsilon^{2 / 3} D^{5 / 3}}{12 \sigma}
$$

where the following relation proposed by Batchelor [2] applies:

$$
\overline{\Delta u^{2}}(D)=\beta(\varepsilon D)^{2 / 3} .
$$

In equation (3), $\varepsilon$ is the mean dissipation rate of turbulent kinetic energy and $\beta$ is a numerical constant obtained by integrating over the whole range of turbulent scales which, according to Batchelor, takes the value $\beta=9 / 5 \Gamma\left(\frac{1}{3}\right) \alpha$ with $\alpha$ a universal constant.

The idea of the existence of a critical Weber number $W e_{c}$, such that droplets and/or bubbles whose $W e>W e_{c}$ are unstable and eventually break up in a given time, is commonly accepted. Unfortunately, in order to fully understand the physical processes where turbulent break-up of particles is a key factor, knowledge of the critical Weber number is clearly insufficient. Therefore, more detailed information including the break-up rate of particles, mean number of fragments produced in a break-up event and the size distribution of those fragments is required

To study the evolution of a population of particles, a number density function, $p(D, \bar{v}, \vec{x}, t)$, which represents the number of particles of a given size $D$ and velocity $\vec{v}$ per unit volume of fluid, is commonly employed. The evolution of $p(D, \bar{v}, \bar{x}, t)$ is given by the following Boltzmann-type equation [22]:

$$
\frac{\partial p}{\partial t}+\nabla_{x} \cdot(\vec{v} p)+\nabla_{z} \cdot(\bar{F} p)=-\frac{\partial(R p)}{\partial D}+\dot{q}_{\mathrm{B}}+\dot{q}_{\mathrm{D}}+\Gamma,
$$

where $\dot{q}_{\mathrm{B}}$ and $\dot{q}_{\mathrm{D}}$ stand for the birth and death rate of particles due to breakage and coalescence, $\Gamma$ accounts for the changes in momentum distribution due to collisions between particles and $R(D)$ is the growth rate of the particle size due to evaporation and dissolubility effects. Equation (4) can be integrated in the velocity space to give

$$
\begin{gathered}
\frac{\partial n(D, \bar{x}, t)}{\partial t}+\nabla \cdot[\bar{U}(D, \bar{x}) n(D, \bar{x}, t)] \\
=-\frac{\partial(R(D) n(D, \bar{x}, t))}{\partial D}+\dot{Q}_{\mathrm{B}}+\dot{Q}_{\mathrm{D}}
\end{gathered}
$$

where $\dot{Q}_{\mathrm{B}}=\int \dot{q}_{\mathrm{B}} \mathrm{d} \vec{v}$ and $\dot{Q}_{\mathrm{D}}=\int \dot{q}_{\mathrm{D}} \mathrm{d} \bar{v}$. Equation (5) is usually called the population balance equation (pbe) and describes the spatio-temporal evolution of the number density of particles, $n(D, \bar{x}, t)$. When the only changes in the population of particles are caused by break-up, the equation simplifies to [15]

$$
\begin{aligned}
& \frac{\partial n(D, \bar{x}, t)}{\partial t}+\nabla \cdot[\vec{U} n(D, \vec{x}, t)] \\
& =\int_{\mathrm{D}}^{\infty} m\left(D_{0}\right) f\left(D, D_{0}\right) g\left(D_{0}\right) n\left(D_{0}, \vec{x}, t\right) \mathrm{d} D_{0} \\
& \quad-g(D) n(D, \bar{x}, t),
\end{aligned}
$$

where $g(D)$ is the break-up frequency, $m\left(D_{0}\right)$ the number of fragments resulting from the break-up of a particle of diameter $D_{0}$, and $f\left(D, D_{0}\right)$ the size probability density function (pdf) of the fragments, commonly named daughter particle pdf. These three functions completely determine the break-up process.

Several break-up models have been developed in the past to describe the turbulent break-up of particles based on the idea of competition between turbulent and surface tension stresses. A comprehensive study of the existing turbulent break-up models can be found in a review by Lasheras et al [11]. Some authors have proposed models based on kinetic theory where the break-up of a particle is caused by particleeddy collisions $[5,13,17,20]$. In all these models the breakup is assumed to be binary. On the other hand, Konno et al [10] proposed a tertiary break-up model in which a mother drop is supposed to be composed of 'unit volumes' Furthermore, the resulting daughter droplets are composed of an integer number of those volumes. The size distribution of the daughter particles minimizes the total energy, and the number of tragments is chosen to be three by best fitting the available experimental data. More recently, Martínez-Bazán et al $[15,16]$ developed a binary model based on dimensional and energy considerations under which the break-up frequency of bubbles, $g$, is given by the inverse of the characteristic time, scaled with the bubble diameter $D$, the continuous phase density $\rho$ and the difference between the turbulent and the surface tension stresses $\Delta \tau$,

$$
g(D) \propto \frac{\sqrt{\Delta \tau / \rho}}{D} .
$$

The daughter size pdi is derived by minimizing the surface energy of the resulting fragments.

Detailed examination of the above models reveal that they disagree in various points, namely:

(i) Number of fragments resulting from the break-up process, $m\left(D_{0}\right)$.

(ii) Dependence of the break-up frequency, $g\left(D_{0}\right)$, on the particle diameter.

(iii) Shape of the daughter particle pdf, $f\left(D, D_{0}\right)$. While some authors propose models where the pdi follows a $\cup$-shaped curve, other models exhibit a $\cap$-shaped curve.

The main purpose of the present work is to provide an experimental technique to obtain reliable experimental data of the turbulent break-up process and, consequently, to validate future and existing closure models. Since the new technique has been applied to study the turbulent break-up process of air bubbles, in the rest of the paper we will refer only to bubbles rather than particles in general.

Keeping in mind these ideas, this paper is organized as follows: section 2 is devoted to the development of the experimental and image processing techniques employed to 


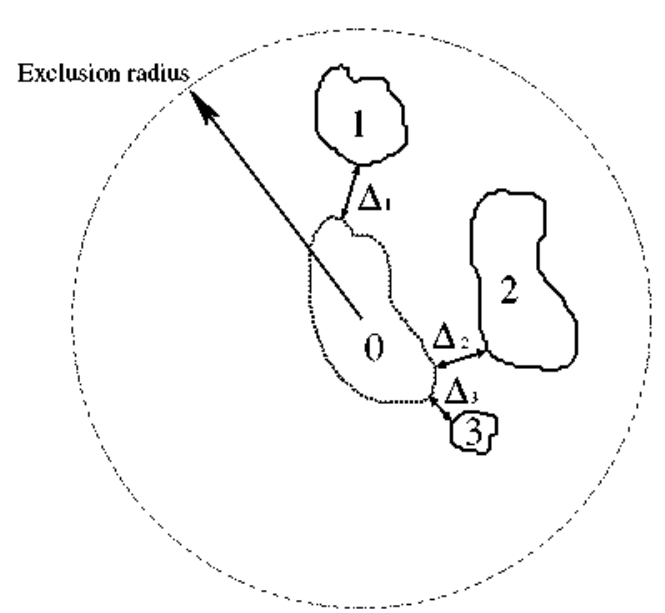

Figure 1. Object in frame $k$ (bubble 0 ) together with the closest objects in frame $k+1$.

obtain the experimental measurements. Section 3 shows some limitations of the tracking technique. The experimental facility is briefly described in section 4 and preliminary experimental results are presented in section 5. Finally, we end with conclusions in section 6 .

\section{Tracking code and statistical data processing}

To study the influence of both the bubble diameter $D_{0}$ and the turbulent dissipation $\varepsilon$ on the bubble break-up, a new computer algorithm has been developed. The program is able to track a large number of bubbles as they are convected downstream by the mean motion of the flow, and automatically detects breakup events. The high degree of automation of this process allows us to analyse the break-up phenomenon statistically for a large number of events and for a wide range of both $D_{0}$ and $\varepsilon$.

The tracking and break-up detection algorithm, hereafter called $t b d a$, is divided into the five following steps:

(i) The first step consists of the detection of the bubbles captured in each frame of the movie. In addition to other parameters of interest, the projected area of every single bubble, $A$, is measured to define its characteristic diameter $D$ as

$$
D=\sqrt{4 A / \pi} .
$$

To detect the bubbles accurately on the image, two different techniques were employed: the first one, called detection by threshold, consisted of simply applying a given threshold level to the images. The pixels belonging to the image of a bubble will have a grey level higher than the applied threshold and, consequently, they will be given the value corresponding to a black pixel. Similarly, those pixels belonging to the image background will have a grey level lower than the applied threshold and they will be given the value conresponding to a white pixel. Therefore, each close group of black pixels can be considered as a bubble. This detection algorithm can be very sensitive to the threshold value selected. Thus, to reduce the sensitivity of the measured bubble area to variations of the illumination conditions, a background subtraction as well as a brightness and contrast enhancement operation were applied to the images before selecting the threshold. More detailed information about the different image processing techniques applied are described in various books related to artificial vision, i.e. [19] and [21] among others.

To reduce the uncertainty of the detection algorithm caused by the selection of the threshold value, a more sophisticated technique, commonly called snakes, was also applied ${ }^{4}$. Although this second technique is more complex than the detection by threshold technique, the perimeters of the bubbles obtained using both methods were found to be almost the same in all the experiments reported.

(ii) Once the bubbles have been detected in each frame, the time evolution of their shape, size and velocity can be described as follows. Since we may encounter several bubbles on a single frame, if we want to describe the time evolution of one of them, we need to distinguish the image of the selected bubble among all the bubbles detected on the following frame. To identify the bubble on the frame $k+1$ which corresponds to the selected one on the previous frame $k$, a pseudo-distance between the image of each bubble in frame $k$, conveniently displaced according to the bubble's velocity (bubble labelled as 0 in figure 1), and the rest of bubbles in the following frame $(k+1)$ is calculated and minimized. Thus, the pseudo-distance function $\Delta_{j}^{*}$ is defined as

$$
\Delta_{j}^{* 2}=\Delta_{j}^{2}+K_{A}\left(\frac{A_{j}-A_{0}}{A_{0}}\right)^{2},
$$

where $j=1, \ldots, J$ is the number of bubbles detected on the frame $k+1, \Delta_{j}$ is the minimum distance between the pixels defining the contour of the selected bubble, labelled as 0 , and the pixels defining the contour of bubble $j$ (see figure 1 ), $A_{j}$ is the projected area of bubble number $j$ and $K_{A}$ is a constant of order one.

This correlation method is based on the assumption that the bubble we are tracking must be close to an expected position, estimated from the bubble's velocity already measured from previous frames. Unfortunately, since, in a turbulent flow, the actual motion of the bubbles is stochastic, it may happen that a bubble different from the selected one may be closer to the estimated position than the one we are tracking. To avoid this type of error in our tracking algorithm, the proposed pseudo-distance includes a term which accounts for changes of some properties of the bubble. Although alternative definitions of the pseudodistance may be employed, one of the properties which has given successful results is a function which depends on the projected area, $\left(A_{j}-A_{0}\right)^{2} / A_{0}{ }^{2}$. Notice that bubbles whose shape is considerably different from that of the selected bubble will increase their pseudo-distance $\Delta_{j}^{* 2}$ and most likely will not be chosen as the tracked bubble. In addition, as shown in figure 1 , an exclusion radius is defined to limit the geometrical searching distance $\Delta_{j}$ and, consequently, to avoid enroneous connections of bubbles far apart from each other.

(iii) The above algorithm by itself is enough to track the bubbles and detect if, at some point, the images of two bubbles

4 More detailed information regarding the shakes technigue can be found in [4]. 
overlap or, more importantly, if they collide due to the turbulent motion of the flow. On the contrary, since in the present algorithm only one object is associated with the bubble we are tracking, a new routine must be included to detect break-up events. The routine included to detect the splitting of a bubble simply consists of the backward application of the same correlation method used to track a bubble. In other words, it consists of searching in frame $k$ for the bubble which corresponds to the selected bubble in frame $k+1$. Thus, if several objects of frame $k+1$ were associated with the same one on frame $k$, it would mean that a break-up event may have happened. Moreover, to validate the break-up event, it must be checked that the presumed mother bubble, encountered on frame $k$, is also associated with at least one of the possible fragments resulting from its break-up on frame $k+1$. For example, if bubbles 2 and 3 in figure 1 are associated with bubble 0 and, at the same time, bubble 0 is associated with either bubble 2 or bubble 3 , the algorithm will determine that bubble 0 has been broken, giving birth to bubbles 2 and 3 on the following image. Other possible situations have also been considered in the algorithm. Examples of different situations where direct application of the steps described so tar works well have been sketched in figure 2 . In this figure, case 1 is the most likely to happen where no break-up or overlap occurs and the bubbles are simply tracked as they move. Notice that bubble $A$ is associated with bubble $a$ and vice versa, while bubbles $B$ and $b$ are associated with each other. On the other hand, case 2 represents a typical break-up event. Here, it can be observed that the mother bubble $B$ splits into daughter bubbles $b$ and $c$. Since both fragments point to bubble $B$ and, at the same time bubble $B$ points to either $b$ or $c$, the $t b d a$ would detect the break-up event. Similarly, situation 3 shows a detected coalescence or overlapping event. Thus, to validate a break-up event, it must be checked that the presumed mother bubble, encountered on frame $k$, is also associated with at least one of the possible fragments resulting from its break-up on frame $k+1$.

Situations 1 and 2 in figure 3 show two cases where the simple bubble association criterion would fail and the backward application is required. This type of misassociation is easily corrected by enforcing each bubble to point to one of the bubbles which point to it. For example, in situation 1 , since bubble $a$ is pointing to bubble $A$ and bubbles $B$ and $b$ are pointing to each other, $A$ would be enforced to point to bubble $a$, being the right association. Other kinds of pathological situations are presented in cases 3 and 4 . Case 3 shows a situation where bubble $B$ breaks into bubbles $b$ and $c$, while a break-up event with bubble $A$ splitting into $a$ and $b$ would be detected by the $t b d a$ technique. These cases are more complicated to determine and, when detected, no possible correction can be easily implemented, so bubbles implicated in the event are simply excluded from the statistics. The detection of these situations is performed by checking the changes in the volume conservation parameter (VCP), as explained below in step (iv).

Finally, although there are methods to distinguish overlapping from coalescing bubbles $[3,18]$ (situations sketched in case 3 of figure 2 and case 4 of figure 3 ), it has been observed that they do not work properly for high Weber number bubbles which are highly distorted and their shape is far from being spherical. Therefore, bubbles which overlap are excluded from statistical computations in our code. In addition, since the tracking code computes the statistics of breaking bubbles by following a single bubble from the moment it is introduced into the flow until the time it breaks up, it is reasonable to take into account only bubbles which do not overlap with each other during their lifetime.

(iv) A fourth step, based on the conservation of volume law, is applied to improve the performance of the $t b d a$. Thus, a VCP for every break-up event is defined as

$$
\mathrm{VCP}=\frac{\sum_{j=1}^{m} A_{j}^{3 / 2}}{A_{0}^{3 / 2}}-1,
$$

where $m$ is the number of daughter bubbles produced after a break-up event, $A_{j}$ the projected area of the $j$ th tragment and $A_{0}$ that of the conresponding mother bubble. In an ideal break-up process where both the mother and the daughter bubbles remained spherical, the VCP would vanish. Although bubbles are far from being spherical in a real break-up event, VCP has been shown to oscillate around a small negative value (see figure 4). Notice that, in figure 4, where the VCP frequency histogram has been plotted for 50 break-up events, the values of VCP range from approximately -0.6 to 0 . The reason why the mean VCP is negative is that, when a bubble is about to break up, it becomes very elongated and, therefore, the volume obtained using the characteristic diameter defined in equation (8) is overestimated. Calibration of the tracking code has shown that most of the spurious break-up events have VCP values about one unit above or below the mean VCP of the correct events. Consequently, lower and upper limits for the VCP can be defined, excluding those events with VCP values outside of these bounds from further computations.

(v) The information collected for each bubble using the tracking code includes the bubble's lifetime, mean velocity, number of fragments produced after the break-up event as well as the size of those fragments. Using the collected data, the above-mentioned closure functions, i.e. $f\left(D, D_{0}\right), g\left(D_{0}\right)$ and $m\left(D_{0}\right)$, can be measured as follows:

- The number of fragments $m$ is measured directly, and consequently a mean value can be obtained for each bubble size, $D_{0}$

- The daughter bubble pdf $f\left(D, D_{0}\right)=f^{*}\left(D / D_{0}\right) / D_{0}$ of the fragments can also be obtained by calculating the observed probability of a given size $D$ to be generated from the break-up of a bubble of diameter $D_{0}$.

- Measurements of the break-up frequency need a little more analysis. To proceed with the analysis, let us consider $N(D, x)$, the number of bubbles of size $D$ that, entering the measuring window at $x=x_{w}$, arrive at a given downstream position $x$ within a fixed measuring window. Since $N(D, x)$ does not account for those bubbles generated due to the break-up of larger 
(1)

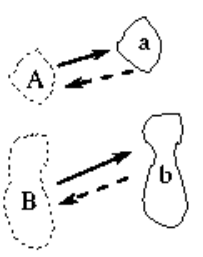

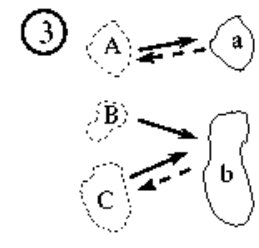

Figure 2. Situations in which the direct application of the $t b d a$ wotks well.
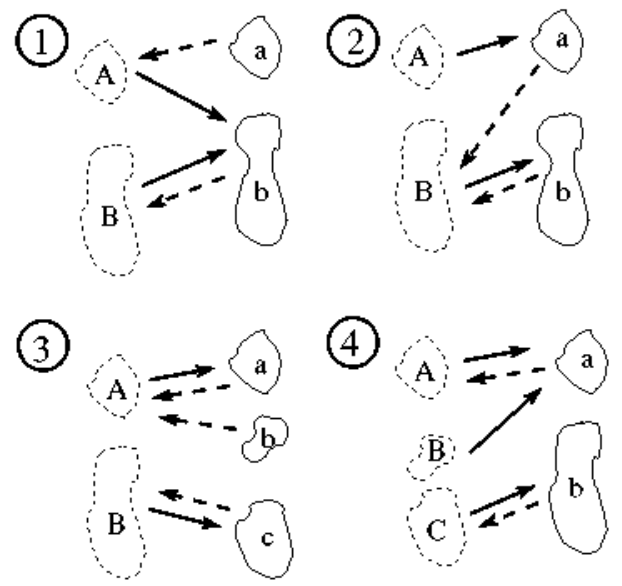

Figure 3. Situations in which the direct application of the $t b d a$ fails and additional work is required.

bubbles, application of equation (6) to $N(D, x)$ simplifies to

$$
g(D, x)=-\frac{1}{N(D, x)} \frac{\partial(U(D, x) N(D, x))}{\partial x} .
$$

Notice that the right-hand side of equation (11) depends only on variables already measured with the tracking algorithm. Therefore the break-up frequency can be obtained for each bubble size as a function of the downstream position.

To conclude the presentation of the algorithm, a brief description of the limitations of the technique is included in the following section.

\section{Limitations of the particle tracking and break-up detection algorithm}

In this section we will mainly analyse the limitations of the code in terms of the errors produced by processing a twodimensional image of the three-dimensional bubble and the maximum number of bubbles that the program can handle.

Although equation (8) is exact enough to measure the size of spherical bubbles whose projected area encloses more than 10 pixels (see [14]), the accuracy of the measurement of $D$ decreases when the bubbles are not spherical. The error caused by the shape of the bubbles can be roughly estimated by considering the bubbles to be elliptical with an aspect ratio given by $\Lambda=a / b$, a situation depicted in figure 5 . In the case shown here the camera would detect a bubble whose major and minor axes are $a$ and $b$ respectively. For the limiting cases $\theta=0$ and $\pi / 2$, the measured sizes are

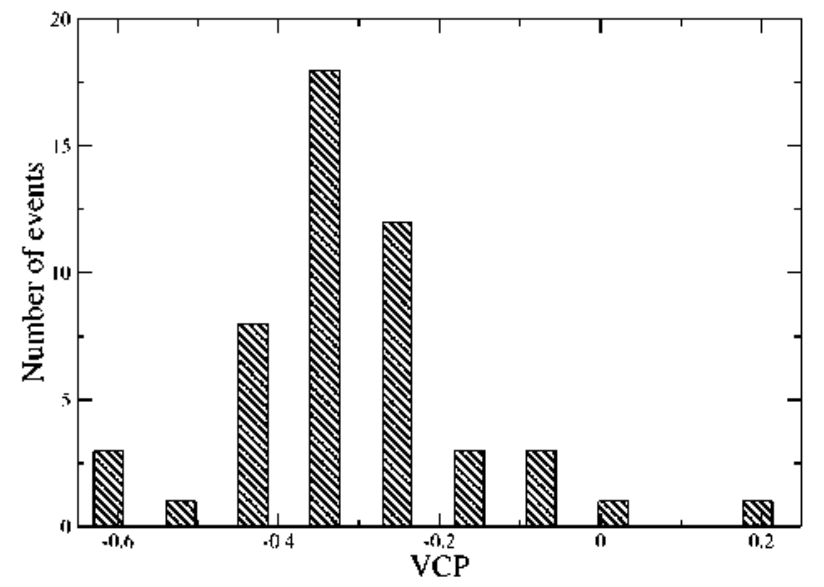

Figure 4. Frequency histogram of the VCP measured for 50 break-up events.

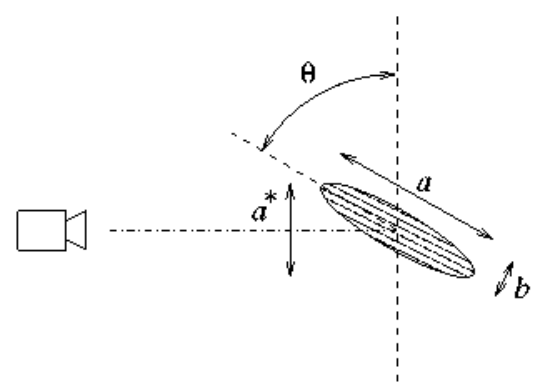

Figure 5. Sketch of an ellipsoidal bubble with an elevation angle $\theta$ with respect to the image plane.

$D_{\theta=0}=2 b \sqrt{\Lambda}$ and $D_{\theta=\pi / 2}=2 b$, while the real value of the equivalent size is $D_{T}=\sqrt[3]{6 \text { volume } / \pi}=2 b \sqrt[3]{\Lambda}$. Therefore, the measured size is bounded by $\Lambda^{-1 / 3}<D / D_{T}<\Lambda^{1 / 6}$. The maximum observed elongation of the bubbles measured in the experimental cases presented in section 5 is $\Lambda=2.7$, giving an error of the measured equivalent diameter which ranges from $0.71<D / D_{T}$ to $1.18>D / D_{T}$. Fortunately, only a very few bubbles exhibit such an extreme aspect ratio, $\Lambda^{*}=a^{*} / b=1.3$ being the most probable one. Furthermore, in an isotropic turbulent flow there is no preferential alignment of the bubbles and the camera will capture their projected image with the same probability for any elevation angle $\theta$. Thus the actual elongation $\Lambda$ can be related to the observed one $\Lambda^{*}$ by

$$
\Lambda^{*}=\frac{2}{\pi} \int_{0}^{\pi / 2} \sqrt{\sin ^{2} \theta+\Lambda^{2} \cos ^{2} \theta} \mathrm{d} \theta,
$$

giving a most probable corrected aspect ratio which takes the value $\Lambda \simeq 1.6$. For this most common case, the error in the measurement of $D$ is bounded by $0.85<D / D_{T}<1.08$.

The present algorithm is meant to be used for dilute twophase flows. An estimation of the maximum concentration of bubbles that the detection technique can process may be obtained by calculating the probability of encountering overlapping bubbles in a single image. Thus, the probability of finding two overlapping bubbles, $P_{0}$, may be given by (Lecuona et al [12]) 


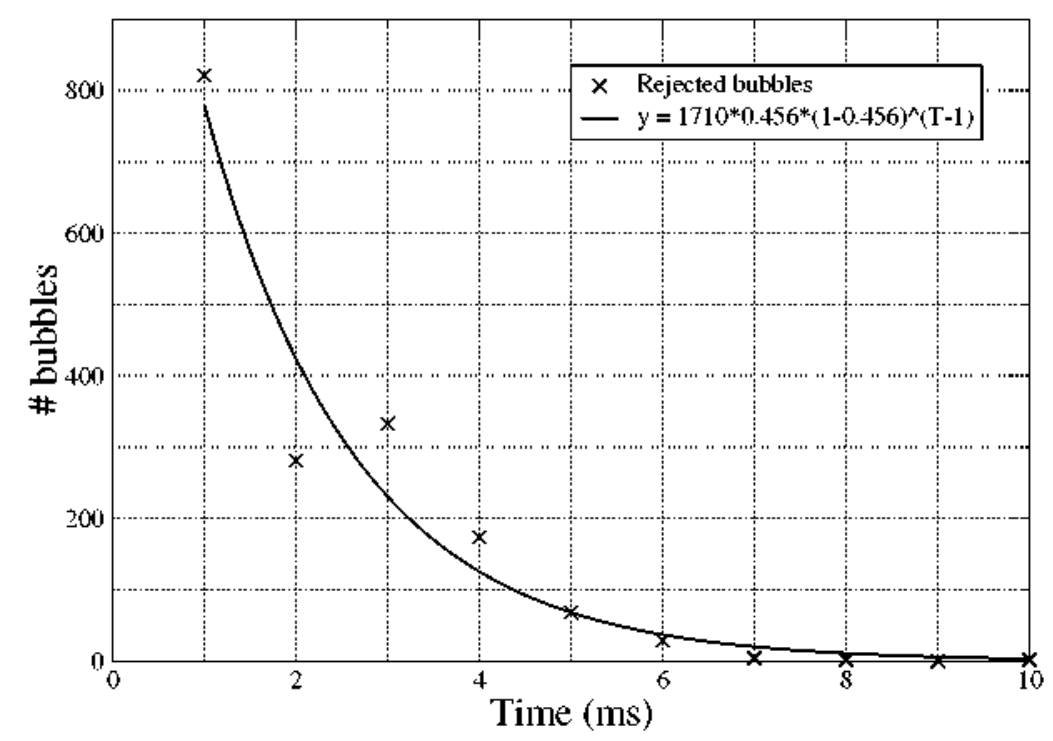

Figure 6. Number of bubbles rejected by the $t b d a$ compared with the predicted value given by equation (16).

(This figure is in colour only in the electronic version)

$P_{\mathrm{o}}=\int_{D_{\min }}^{\infty} \int_{D_{\min }}^{\infty}\left[1-\exp \left(4 \pi \lambda z D_{1} D_{2}\right)\right] p\left(D_{1}\right) p\left(D_{2}\right) \mathrm{d} D_{1} \mathrm{~d} D_{2}$

where $D_{\text {min }}$ is the minimum bubble size accurately resolved by the imaging technique, $p(D)$ is the bubble size pdf, $\lambda$ is the number of bubbles per unit volume of the flow and $z$ is the image depth of field, i.e. the depth of the region where bubbles are in focus. Since in our particular application the depth of field was always larger than the diameter of the bubbly jet, $z$ will be taken to be the jet's diameter. Moreover, considering that the measured bubble size distribution follows an exponential decay for bubble sizes larger than the most probable one,

$$
p(D)=\frac{1}{\mathcal{D}} \exp \left(-\frac{D-D_{\min }}{\mathcal{D}}\right)
$$

equation (13) can be integrated to yield

$$
\begin{gathered}
P_{0}\left(\xi, D_{\min } / \mathcal{D}\right)=1-\frac{1}{\xi} \exp \left(\frac{1}{\xi}+2 \frac{D_{\text {nin }}}{\mathcal{D}}\right) \\
\times \int_{\frac{1}{\xi}\left(1+\xi \frac{\left.\min _{2}\right)^{2}}{\infty}\right.}^{\infty} \frac{\exp (-t)}{t} \mathrm{~d} t
\end{gathered}
$$

where $\xi=4 \pi \lambda z \mathcal{D}^{2}$ is a measure of the concentration of bubbles. Once the probability of a bubble overlapping with another one in a single image is known, we can compute the probability of a bubble to be rejected in frame number $T$ of the video or, in other words, the probability of a bubble to live during $T-1$ frames without overlapping, as

$$
P(T)=\left(1-P_{0}\right)^{T-1} P_{0}
$$

To test the applicability of equation (16) we have obtained the number of bubbles rejected in the experimental set 2 , described in section 5, and compared it with the prediction given by equation (16). Analysis of the video images yields the following parameters to be included in expressions (13)-(16):
$D_{\text {min }}=0.4 \mathrm{~mm}, \mathcal{D}=0.852 \mathrm{~mm}, \lambda \simeq 0.002$ bubbles $\mathrm{mm}^{-3}$ and $z \simeq 20 \mathrm{~mm}$. Thus, under the above conditions, the probability of finding two overlapping bubbles in a single image is $P_{\circ}=0.45$.

Figure 6 shows the number of bubbles rejected versus their lifetime for the experimental set 2 described in section 5 . The total number of rejected bubbles is $N_{\mathrm{t}}=1714$. Note that the prediction of the number of bubbles rejected after $T$ frames, $N_{\mathrm{r}}(T)=N_{\mathrm{t}}\left(1-P_{0}\right)^{T-1} P_{0}$, is in very good agreement with the experimental results, therefore showing the efficiency of the tbda in detecting overlapping bubbles. The probability of finding two overlapping bubbles as a function of the concentration of bubbles is shown in figure 7 for two different values of the minimum bubble size resolved.

\section{Experimental facility}

To investigate the performance of our $t b d a$ algorithm experimentally, we decided to analyse the influence of the surrounding flow on the deformation and break-up of a bubble. Therefore, we selected a turbulent water jet, generated by discharging a jet of water upwards through a nozzle located at the bottom of an acrylic tank of square cross-section (see figure 8) as our turbulent base flow. To minimize the recirculating flow produced in the tank by the high-momentum jet, the water injected was allowed to overflow from the top of the tank through a set of gutters placed on each side. A piece of honeycomb and a mesh were installed inside the nozzle, which, together with the high contraction ratio (70:1), ensured a uniform velocity profile at the nozzle exit. The diameter of the exit nozzle, $d=6 \mathrm{~mm}$, used in all experiments reported here, was much smaller than both the width of the cross-section ( $L_{\text {tatk }}=0.5 \mathrm{~m}$ ) and the height $\left(H_{\text {tank }}=2 \mathrm{~m}\right.$ ) of the tank, therefore permitting the jet to behave as a fully turbulent, free jet.

The water flow rate of the jet, $Q_{w}$, could be systematically varied from $1.7 \times 10^{-5}$ to $3.4 \times 10^{-4} \mathrm{~m}^{3} \mathrm{~s}^{-1}$ giving exit velocities which ranged from $U_{\mathrm{J}}=0.6$ to $12 \mathrm{~m} \mathrm{~s}^{-1}$. Thus, the 


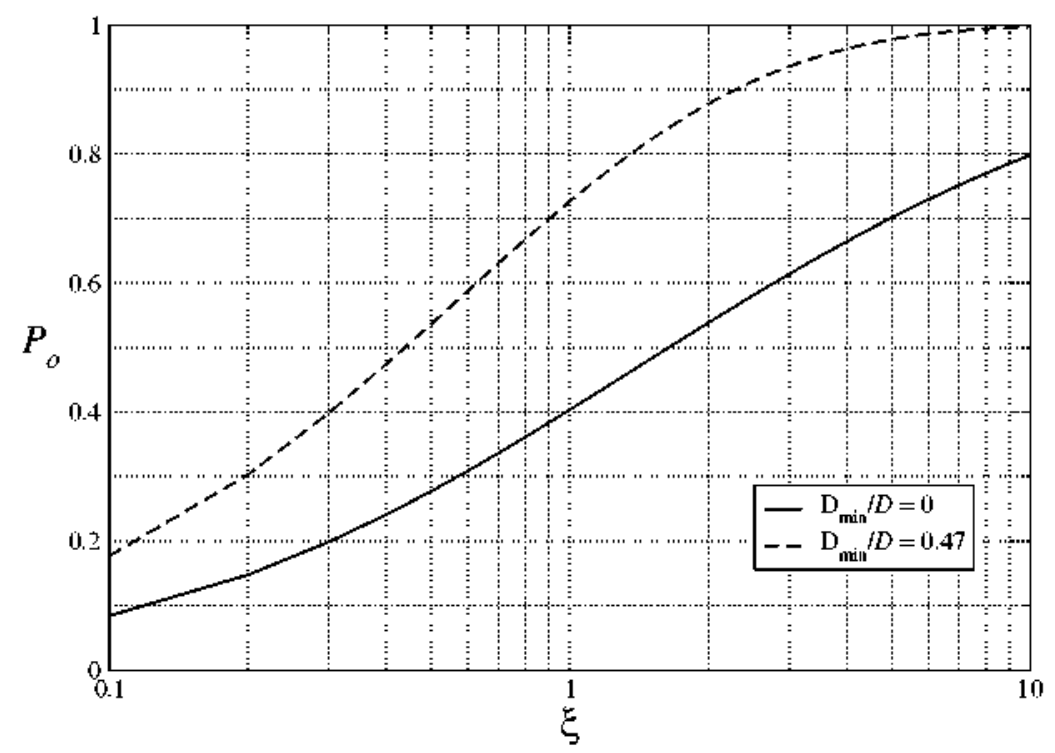

Figure 7. Dependence of the probability of a bubble to overlap, $P_{0}$, on the concentration of bubbles for two values of dimensionless minimum bubble size.

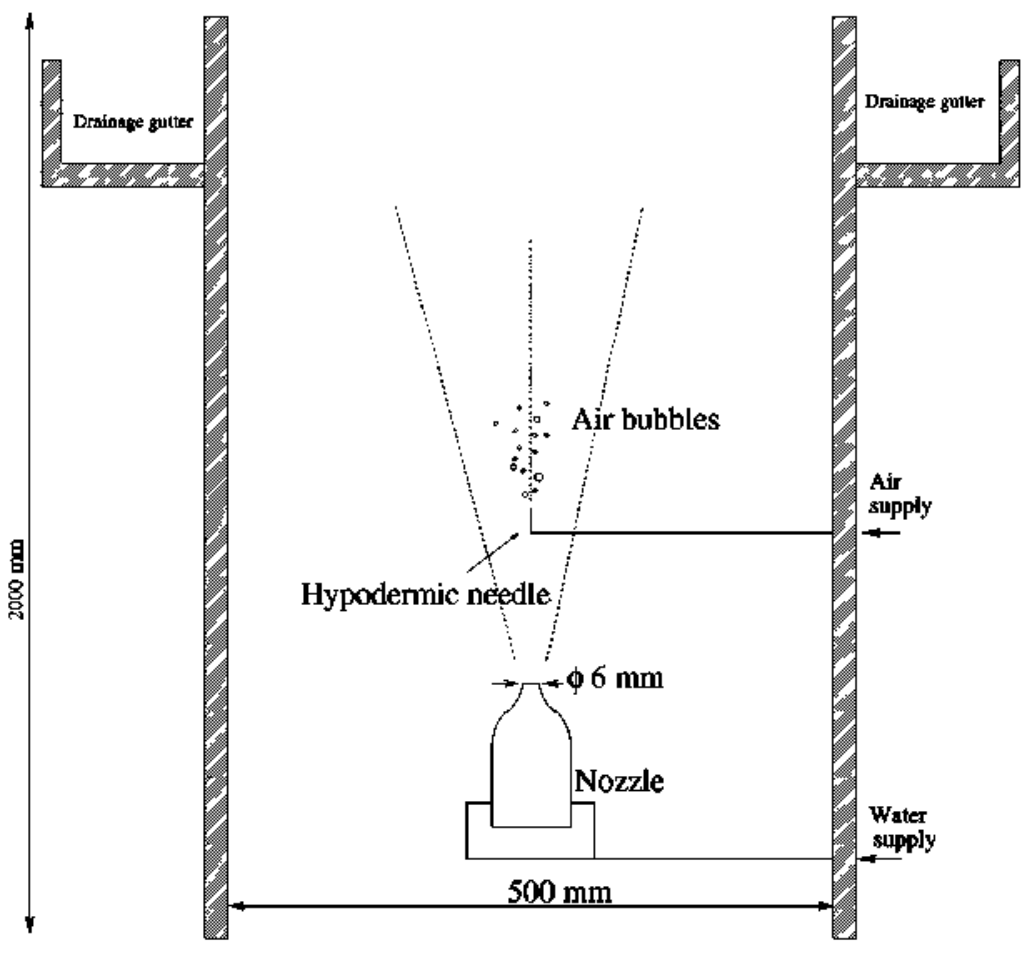

Figure 8. Experimental facility.

jet Reynolds number, $R e=U_{\mathrm{J}} d / v$, could be varied from $R e=$ $3.5 \times 10^{3}$ to $7 \times 10^{4}$, where $v$ is the kinematic viscosity of water.

Bubbles were generated by injecting air from a hypodermic needle along the central the axis of the water jet, in a downstream location far enough away from the exit nozzle to consider the turbulence fully developed $(x / d>15)$. Under these conditions, when both the jet Reynolds number and the turbulent Weber number, given by equation (2), are sufficiently large to neglect viscous effects during the breakup process, the break-up properties of bubbles whose diameter ranges from the Kolmogorov length scale to the integral length scale, $\eta \ll D_{0} \ll \ell$, depend only on the turbulent dissipation rate, $\varepsilon$, and on the bubble diameter, $D_{0}$. Although, at the axis of the jet, the turbulence slowly varies with distance, it can be considered as nearly isotropic and homogeneous within length scales of the order of the size of the bubbles. In all experiments reported here, the air injection velocity was the same as the local jet velocity to prevent shear stresses taking place in the deformation and break-up process. To achieve the above conditions, the air flow rate was varied from $1.7 \times 10^{-8}$ to $2.7 \times 10^{-6} \mathrm{~m}^{3} \mathrm{~s}^{-1}$, depending on the experimental test. In addition, to ensure that the properties of the turbulence 


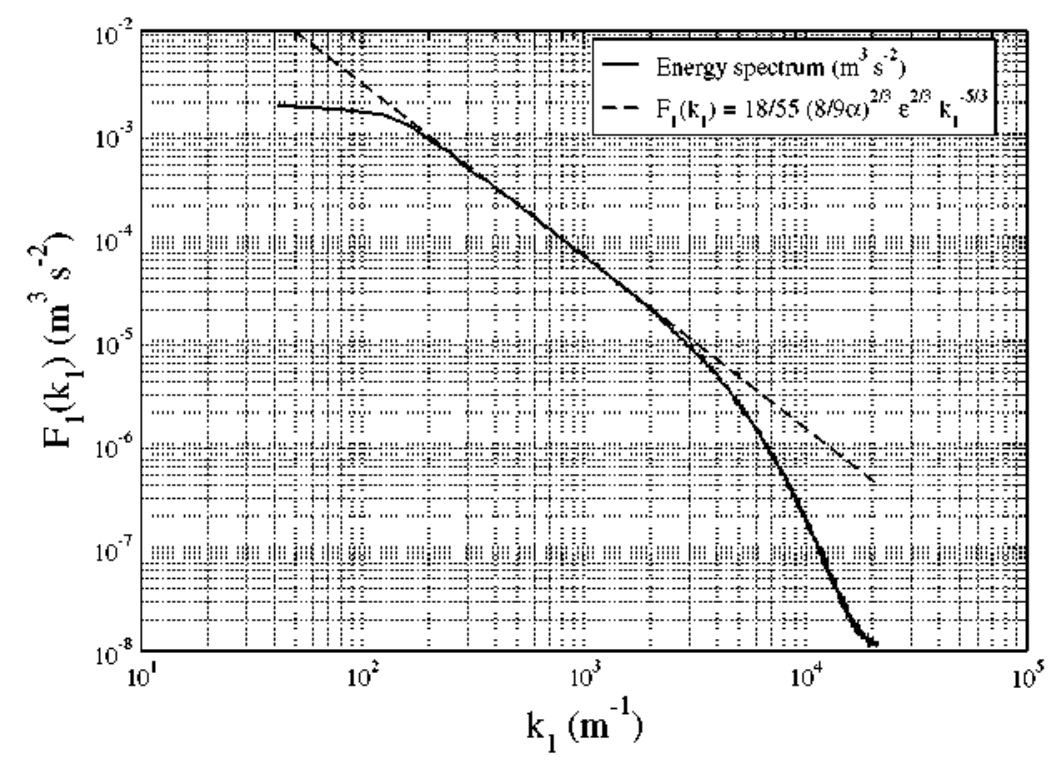

Figure 9. Energy spectrum obtained at $x / d=25, R e=70700$.

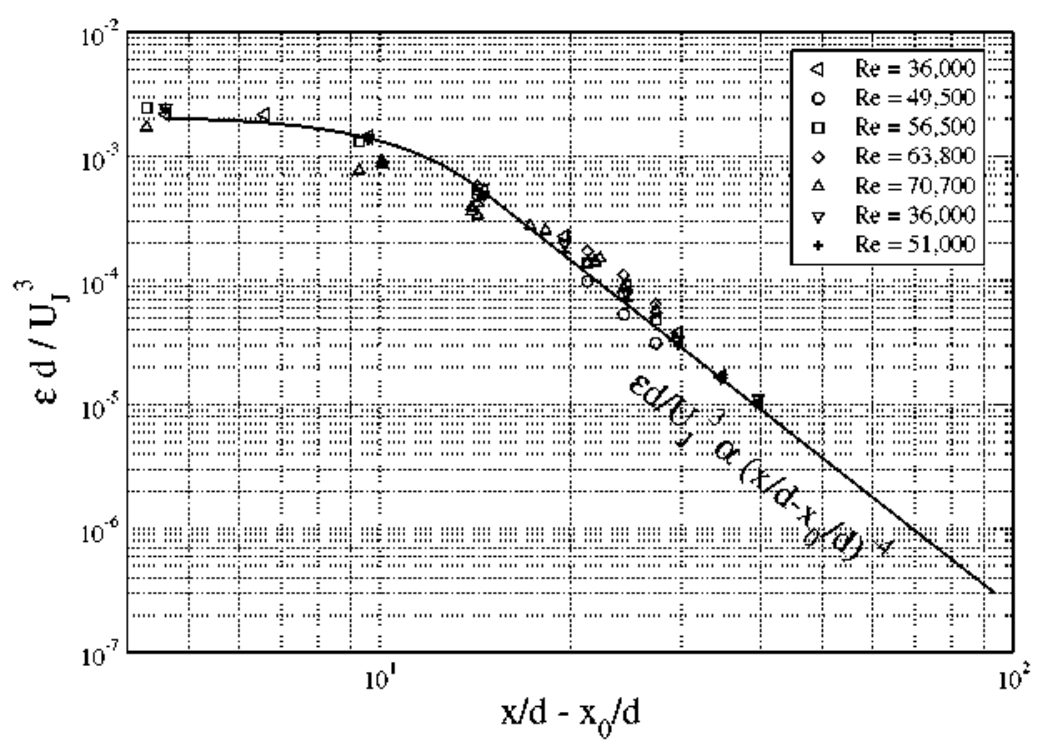

Figure 10. Non-dimensional turbulent dissipation tate as a function of $x / d$ for different values of the Reynolds number, $x_{0} / d=5.76$.

affecting the bubbles were nearly homogeneous and isotropic, we limited our studies to cases where the bubble break-up was completed at a specified downstream distance from the air injection point and where the bubbles remained in the core of the jet (radial distances less than $1 / 3 L_{\mathrm{J}}$, where $L_{\mathrm{J}}$ is the value of the width of the jet at the measuring point).

The turbulent field of the free jet has been widely studied in the past (see $[6,7]$ among others). Thus, it has been reported that, in the far field of the jet, at the central axis, the mean velocity $U_{\mathrm{c}}$ and turbulent dissipation rate $\varepsilon$ are given by

$$
\begin{gathered}
U_{c}(x)=\frac{U_{\mathrm{J}} B}{x / d-x_{0} / d} \\
\varepsilon(x)=\frac{C U_{\mathrm{J}}^{3} / d}{\left(x / d-x_{0} / d\right)^{4}},
\end{gathered}
$$

where $U_{\mathrm{J}}$ is the injection velocity, $d$ is the nozzle's diameter, and $C, B$ and $x_{0}$ are constants to be obtained experimentally.

To determine the above constants and to check that in fact our jet behaves as a high Reynolds number, turbulent, free jet $[1,7]$, we conducted an extensive set of measurements using hot-film anemometry. Figure 9 shows a typical power spectrum together with a dashed line indicating the $-5 / 3$ power law, and inertial sub-range given by [8]

$$
F_{1}\left(k_{1}\right)=\frac{18}{55}\left(\frac{8}{9 \alpha}\right) \varepsilon^{2 / 3} k_{1}^{-5 / 3}
$$

where $\alpha=0.452$ is an empirical constant provided by Gibson [7] for turbulent round jets.

Once the energy spectrum has been measured, equation (19) allows us to obtain the turbulent dissipation rate, $\varepsilon$, at any given location along the central axis. Measurements 


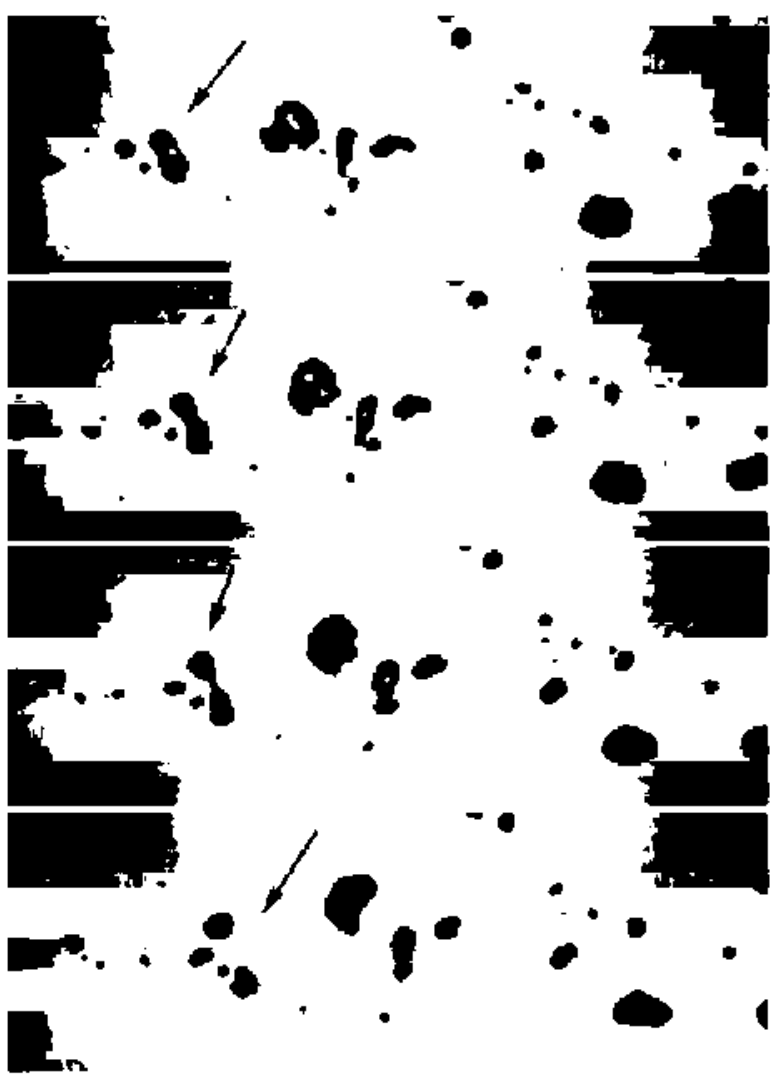

Figure 11. Characteristic break-up sequence.

of $\varepsilon d / U_{\mathrm{J}}^{3}$ are presented in figure 10 for different downstream positions. Notice that for values of $x / d>15$ the turbulent dissipation rate decays with the power law given by equation (18), therefore confirming that our turbulent water jet in fact behaves as a free turbulent jet.

\section{Results}

The spatio-temporal evolution of the bubbles has been recorded at 1000 frames per second, $\Delta t=1 \mathrm{~ms}$, with a Kodak Ektapro high-speed camera. A typical sequence is shown in figure 11 where a break-up event can be observed of a bubble splitting into two daughter bubbles on the fourth frame. The images were stored on a PC for later processing and analysis.

Preliminary results have been obtained with the tbda technique for moderately low values of the turbulent Weber number. The water jet Reynolds number was about $R e=70700$. Four different sets of experiments, whose experimental conditions are given in table 1 , were performed where the air flow was injected at several downstream positions varying from $x_{1} / d=17.5$ to 33 , with $x_{1}$ being the distance from the water nozzle, through a hypodermic needle of diameter $d_{\mathrm{s}}=0.838 \mathrm{~mm}$. The air injection velocity was the same as the local mean water jet velocity at the injection point, $U_{\mathrm{c}}\left(x_{\mathrm{i}} / d\right)$. The measured values of the turbulent dissipation rate varied from $\varepsilon \simeq 12$ to $192 \mathrm{~m}^{2} \mathrm{~s}^{-3}$ giving, therefore, a range of turbulent Weber numbers varying from $W_{e_{t}} \simeq 0.5$ to 3.35 for a mean bubble diameter of $D=1 \mathrm{~mm}$. On the other hand, the Weber number based on the dynamic pressure
Table 1. Experimental conditions.

\begin{tabular}{llllll}
\hline & & $U_{c}\left(x_{1} / d\right)$ & $\begin{array}{c}c\left(x_{\mathrm{i}} / d\right) \\
\left(\mathrm{m}^{2} \mathrm{~s}^{-3}\right)\end{array}$ & \multicolumn{2}{c}{$D=1 \mathrm{~mm}$} \\
\cline { 5 - 6 } Set & $x_{\mathrm{i}} / d$ & $\left(\mathrm{~m} \mathrm{~s}^{-1}\right)$ & $W e_{V}$ \\
\hline 1 & 17.5 & 4.63 & 192 & 3.35 & 0.10 \\
2 & 20 & 3.81 & 124 & 2.53 & 0.26 \\
3 & 24 & 2.98 & 62 & 1.64 & 0.05 \\
4 & 33 & 2.00 & 12 & 0.5 & 0.07 \\
\hline
\end{tabular}

varied from from $W e_{U}=\rho\left(U_{\mathrm{b}}-U_{\mathrm{f}}\right)^{2} D / 2 \sigma \simeq 0.07$ to $W e_{U}=\rho\left(U_{\mathrm{b}}-U_{\mathrm{f}}\right)^{2} D / 2 \sigma \simeq 0.26$ for the same bubble size. Here $U_{\mathrm{b}}$ represents the bubble velocity and $U_{\mathrm{f}}$ is the fluid velocity, taken as the velocity of the smallest bubble measured in the flow. Under the above experimental conditions, the break-up was found to be binary, $m=2$, in all the experiments reported here.

Measurements of the bubble velocity are shown in figure 12. Figure 12(a) shows the downstream evolution of the velocity of $1 \mathrm{~mm}$ diameter bubbles as they move away from the injection needle. It may be observed that, although the air is injected at the same velocity as the water local mean velocity, the air ligament slows down during the detachment process of the bubbles, followed by an acceleration process until they reach the mean fluid velocity. During the acceleration process the velocity of the bubbles differs from that of the fluid, therefore producing a new break-up mechanism driven by the dynamic pressure Weber number, $W e_{U}=\rho\left(U_{\mathrm{b}}-U_{\mathrm{f}}\right)^{2} D / 2 \sigma$ In addition, figure 12(b) shows that the difference between the bubble and the fluid velocity increases with bubble diameter.

The break-up frequency of bubbles of a certain size can be extracted from the Lagrangian approach of equation (11)

$$
g(D, t)=-\frac{1}{N(D, t)} \frac{\partial(N(D, t))}{\partial t} .
$$

To evaluate the right-hand side of equation (20) numerically, the following potential law was fitted to the experimental measurements of $N(D, t)$ :

$$
N_{\text {fit }}(D, t)=a(D) t^{-b, D t} .
$$

Substituting equation (21) into (20) we may obtain the breakup frequency as

$$
g(D, t)=\frac{b(D)}{t}
$$

or equivalently as

$$
g(D, x)=\frac{b(D)}{\int_{x_{1}}^{x} 1 / U(D, s) \mathrm{d} s}
$$

The experimental evolution of the number of bubbles whose diameter is $1 \mathrm{~mm}$ is depicted in figure 13 for the four experimental sets. Notice that in all the cases the number of bubbles decreases with the downstream distance, indicating that, since we are not taking into consideration coalescing bubbles, they are undergoing a break-up process, until they eventually reach a position where no break-up takes place. Application of equations (21) and (22) to the experimental data presented in figure 13 allows us to obtain the break-up frequency as a function of the downstream position. Moreover, since both the mean velocity of bubbles and the downstream 


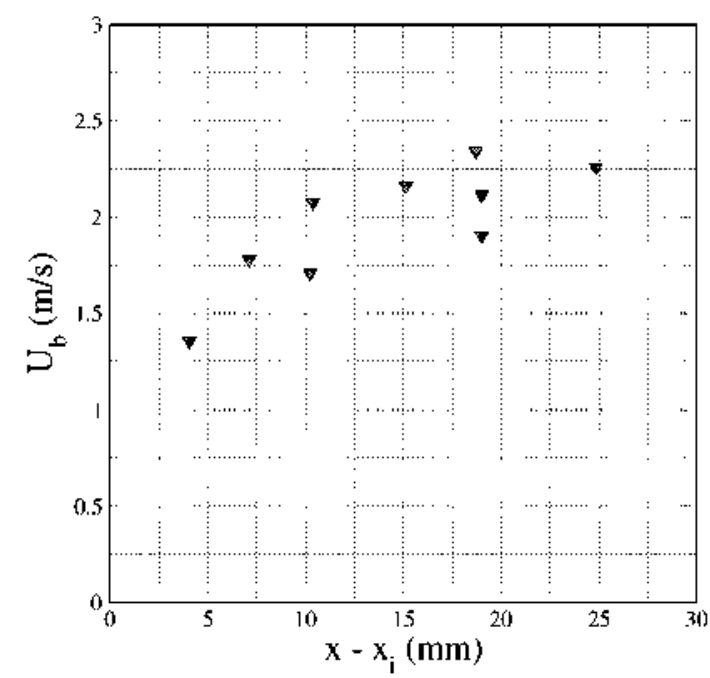

(a)

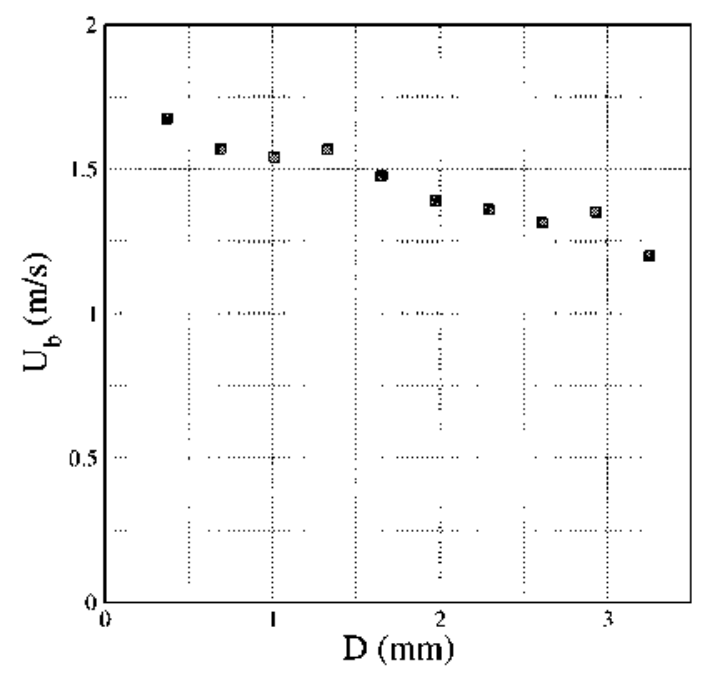

(b)

Figure 12. Measured axial velocity as a function of (a) the downstream distance and (b) the bubble size. The results correspond to experimental set 2 in table 1.

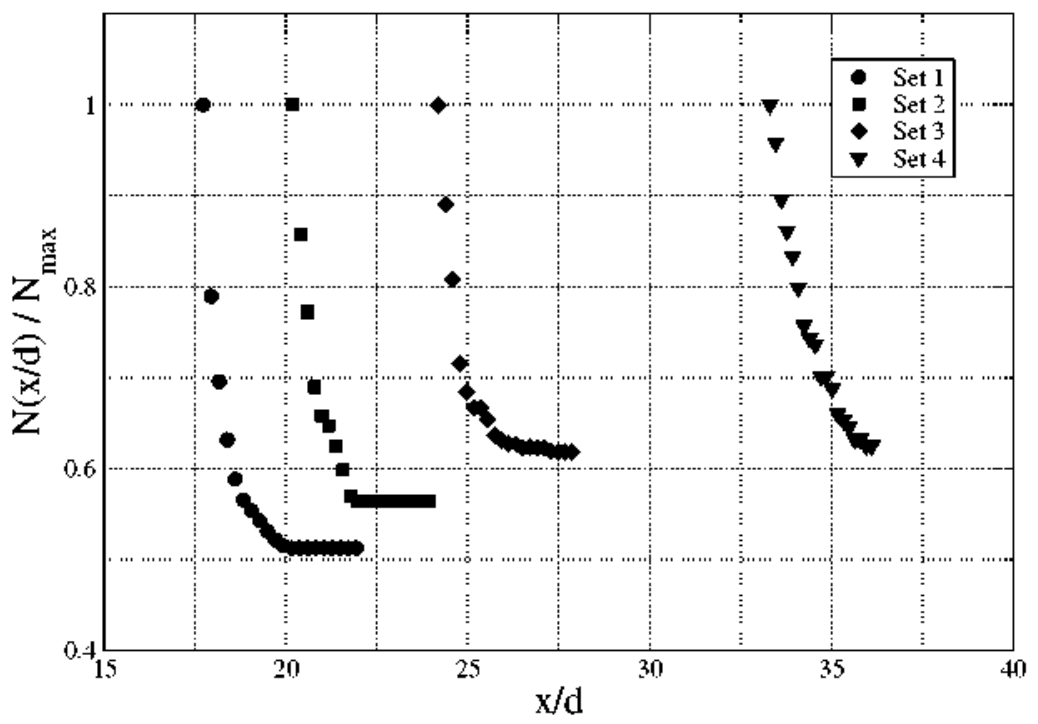

Figure 13. Spatial evolution of the number of bubbles of diameter $D=1 \mathrm{~mm}$ for different experimental conditions.

evolution of turbulent dissipation rate, $\varepsilon(x)$, have already been measured, we can express the measured break-up frequency as a function of $x$ or $\varepsilon$. Thus, the dependence of the break-up frequency on both $x$ and $\varepsilon$ is shown in figures 14(a) and (b) for all the experimental sets and, for clarity, only for bubbles whose diameter is $1 \mathrm{~mm}$. Note that, although the break-up frequency increases with $\varepsilon$, a result in agreement with previous models, it depends on the bubble's time of flight rather than on $\varepsilon$. In fact, it can be observed in figure 14 (b) that, for the same value of $\varepsilon$, the break-up frequency takes different values for bubbles injected at different positions.

In addition to obtaining the dependence of the breakup frequency on $\varepsilon$ and/or $x$, we have also calculated the dependence of the break-up frequency on the bubble diameter $D$. To our knowledge, such a dependence, measured with our bubble tracking algorithm, has never been experimentally reported before. Figure 15 exhibits the dependence of the break-up frequency on bubble diameter. The figure, in agreement with figure 14, shows that, under the experimental conditions given in table 1, the break-up frequency is almost independent of the injection point. This result suggests that, under the low Weber number conditions given in table 1 , the break-up process we are observing is strongly influenced by the stresses suffered by the bubble during the detachment process from the needle.

Thus, if the break-up of bubbles were caused only by the turbulent stresses we could define a dimensionless frequency given by

$$
g^{*}\left(D^{*}\right)=\frac{g(D, x) D^{2 / 3}}{\beta^{1 / 2} \varepsilon^{1 / 3}},
$$

where $D^{*}=D / D_{\mathrm{c}}$ is the dimensionless diameter. To obtain the dimensionless bubble size a critical diameter, whose 


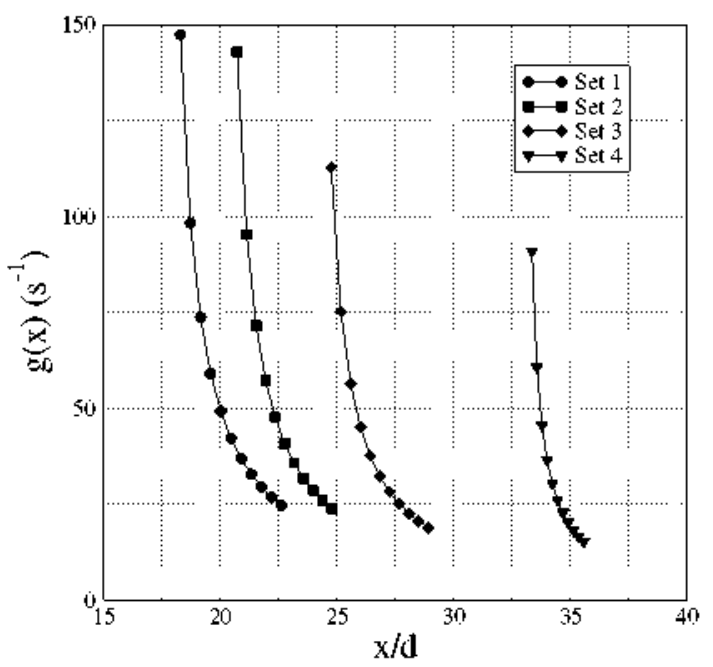

(a)

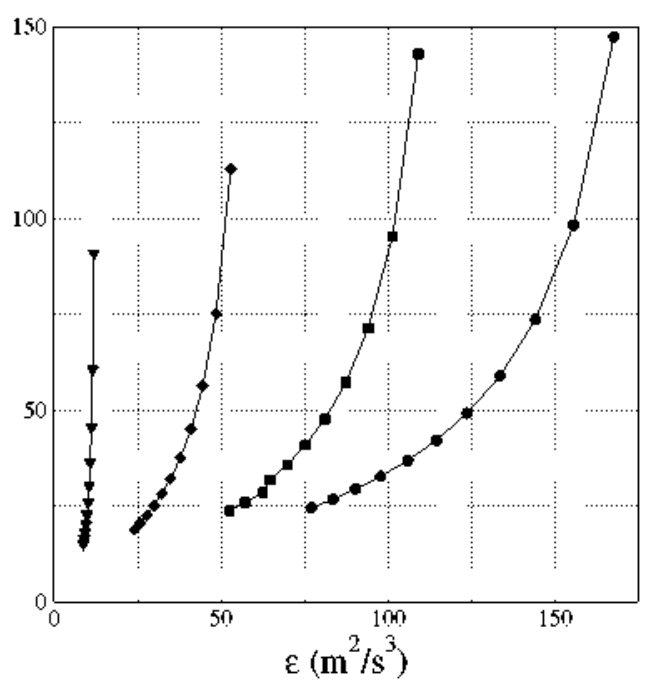

(b)

Figure 14. (a) Break-up frequency for $D=1 \mathrm{~mm}$ bubbles as a function of $x / d$. (b) Break-up frequency as a function of $\varepsilon$ for $D=1 \mathrm{~mm}$ bubbles.

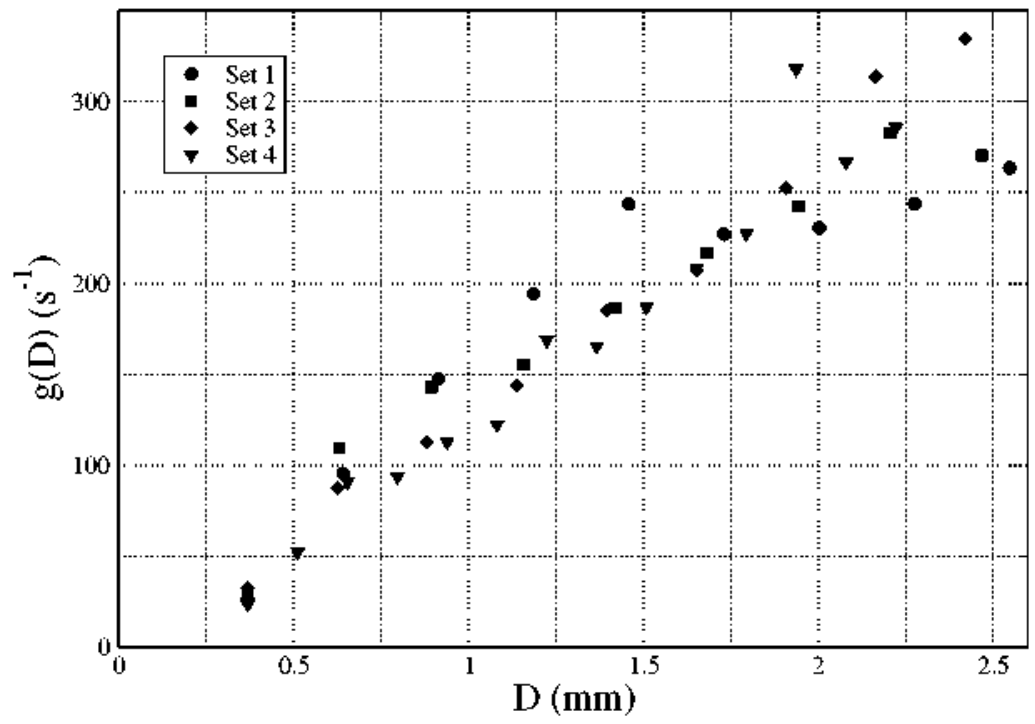

Figure 15. Break-up frequency for $1 \mathrm{~mm}$ bubbles at time $t=2 \mathrm{~ms}$ after injection.

turbulent Weber number is equal to one, has been employed,

$$
D_{\mathrm{c}}=\left(\frac{12 \sigma}{\beta \rho \varepsilon^{2 / 3}}\right)^{3 / 5}
$$

Martínez-Bazán et al [15] proposed a turbulent break-up model given by

$$
g^{*}\left(D^{*}\right)=K_{g} \sqrt{1-1 / D^{* 5 / 3}}=K_{g} \sqrt{1-1 / W e_{\mathrm{t}}} .
$$

Although figure 14 shows that, under the experimental conditions tested here, the break-up frequency does not scale with $\varepsilon$, we may extend equation (26) to more general situations where the break-up process is caused by surface stresses different from the turbulent ones. In particular, if the splitting of the bubbles were caused by any other deforming stresses, the dimensionless break-up frequency would be given by

$$
g^{*}(W e)=K_{1} \sqrt{1-K_{z} / W e},
$$

with $W e=\tau(D) D / \sigma$ and $\tau(D)$ the deforming stresses acting on the surface of the bubble. The dimensionless break-up frequency given by equation (27) is shown in figure 16 . This figure shows that the break-up frequency increases with the Weber number or, similarly, with the dimensionless diameter, until eventually it reaches an asymptotic value given by the constant $K_{1}$. Further experiments are already being performed in our experimental facility at higher values of the Weber number to ensure that the break-up mechanism depends only on the instantaneous flow properties, to determine if $g^{*}(W e)$ follows, in fact, the presumed theoretical model.

In addition to measuring the velocity and the break-up frequency of bubbles, the $t b d a$ algorithm allows us to obtain the size pdf of the daughter bubbles, $f^{*}\left(D / D_{0}\right)$, formed upon the break-up of the given mother bubble. Experimental measurements of the dimensionless daughter size distribution as a function of the dimensionless volume, $V / V_{i}$, obtained for several bubble diameters, are plotted in figure 17 . In this figure 


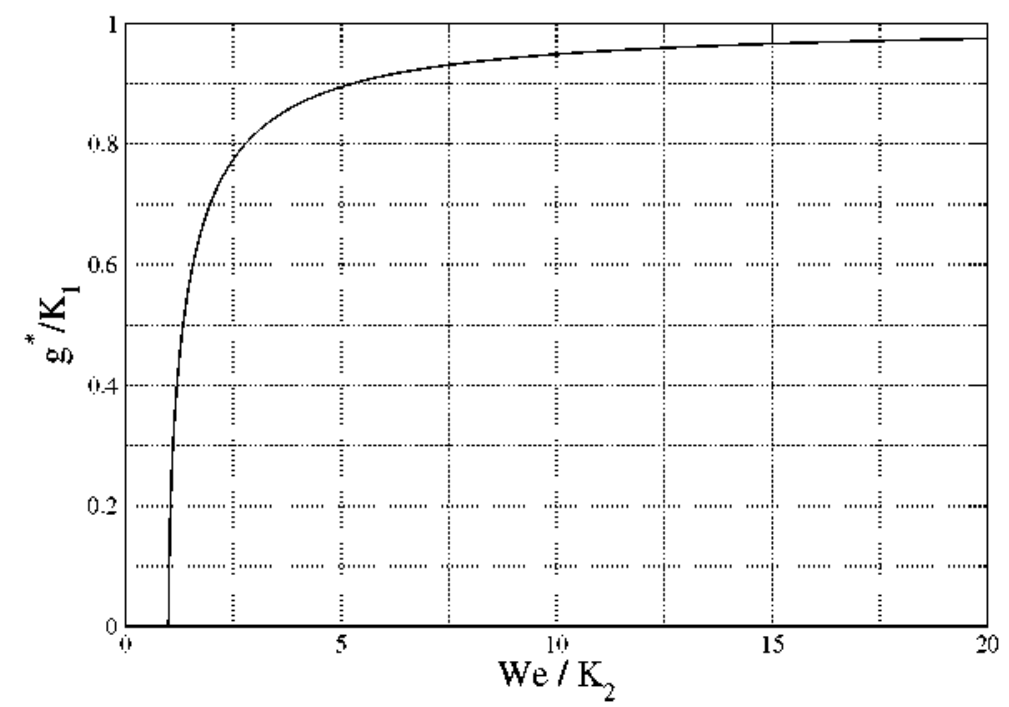

Figure 16. Theoretical dimensionless break-up frequency $g^{*}$ as a function of the Weber number, we.

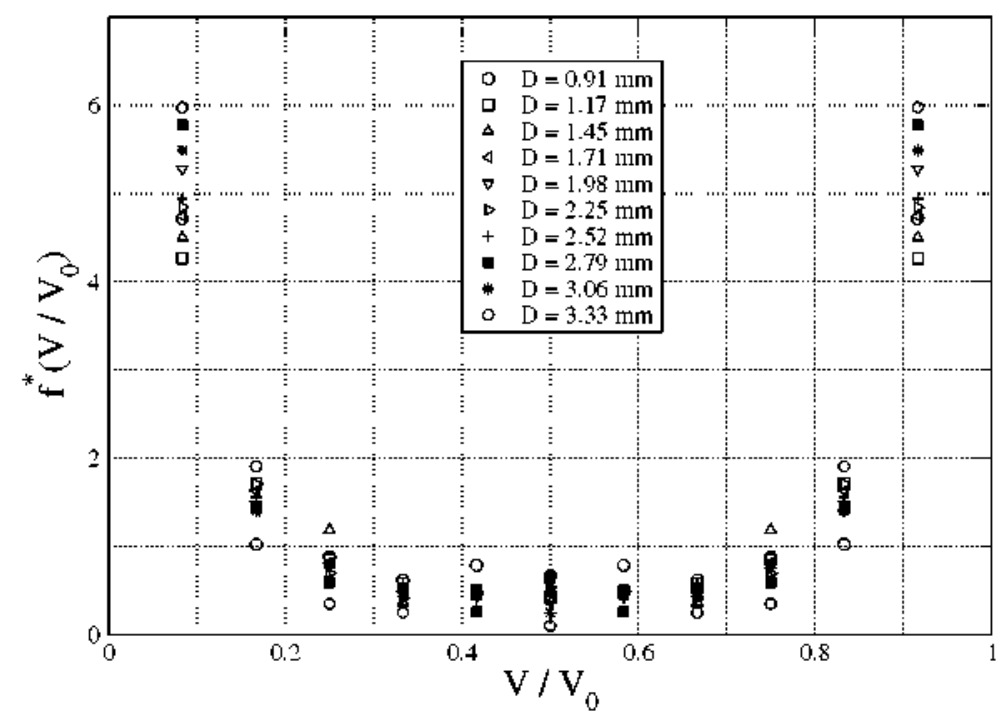

Figure 17. Daughter bubble size pdf obtained for different values of the mother bubble. The experimental results correspond to set 2 in table 1 .

$V / V_{0}$ represents the volume of the daughter bubble formed, divided by the volume of the corresponding mother bubble, $V_{0}$. It may be observed that $f^{*}\left(V / V_{0}\right)$ exhibits a $U$-shape, almost independent of the size of the mother bubble, indicating that the most probable break-up phenomenon corresponds to a binary rupture forming a large and a small bubble. Since $f^{*}\left(V / V_{0}\right)$ does not seem to depend on the mother bubble's diameter, in figure 18 we have plotted the averaged daughter bubble size distribution obtained in each experimental test indicated in table 1 . Notice that the shape of $f^{*}\left(V / V_{0}\right)$ is not only independent of the mother bubble diameter but also independent of the flow conditions under the dynamic pressure conditions investigated in this paper. Therefore, although the break-up model proposed by Tsouris and Tavlarides [20] underestimates the turbulent break-up process taking place under homogeneous and isotropic conditions, it seems to give qualitatively good results in flows where both phases move at different velocities, i.e. shear flows.

\section{Conclusions}

A simple, novel tracking technique has been developed to investigate the break-up properties of particles in turbulent flows. It consists of automatically tracking a large number of bubbles as they are convected by the mean flow, and detecting the break-up events. The tracking code allows us to measure the most relevant characteristics of the break-up process in each event, i.e. the lifetime of the mother bubble, its mean velocity, the number of daughter bubbles and the size of those bubbles among others. Statistical processing of this information provides valuable data to validate the predictions of break-up models.

Although the performance of the $t b d a$ technique is still questionable when the bubble concentration increases considerably, it gives satisfactory results in dilute two-phase flows. Preliminary experimental results, carried out at low values of the bubble Weber number, have been obtained to 


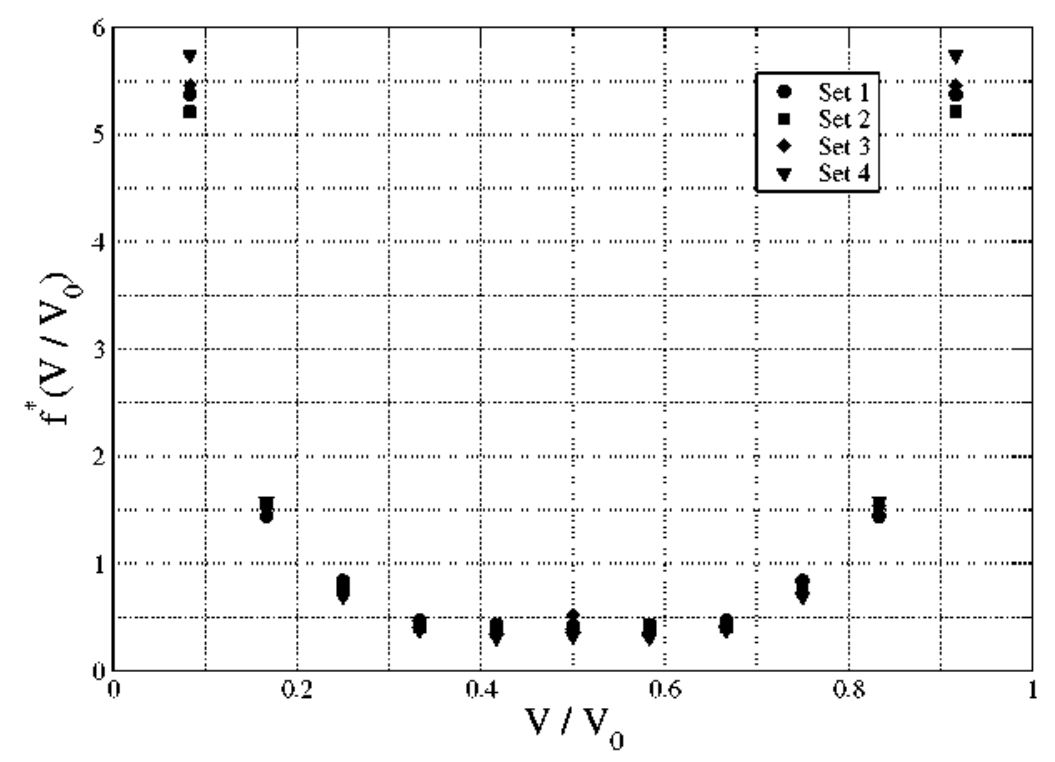

Figure 18. Daughter bubble size pdf obtained for different flow conditions.

demonstrate the performance of the method. The results obtained show an increasing function of break-up frequency with bubble diameter, probably caused by excitation of the natural vibration modes during bubble detachment from the injection needle. Furthermore, measurements of the daughter size distribution exhibit a $U$-shaped behaviour independently of the flow conditions and the mother bubble diameter.

Further experiments at higher values of the bubble Weber number are taking place to determine the dependence of the break-up frequency on the mother bubble diameter, the exact shape of the daughter bubble pdf, $f^{*}\left(D / D_{0}\right)$, and the limitations of the binary break-up assumption.

\section{Acknowledgments}

This research was supported by the Fifth Framework Programme of the European Commission under the Energy, Environment and Sustainable Development Contract No EVG1-CT-2001-00042 EXPRO and by the Spanish MCyT under Projects No PB98-0142-C04-02, DPI2001-4603-E and DPI2002-04550-C07-06.

\section{References}

[1] Antonia R A, Satyaprakash B R and Husain A K M F 1980 Measurements of dissipation rate and some other characteristics of turbulent plane and circular jets Phys. Fluids $23695-700$

[2] Batchelor G K 1953 The Theorv of Homogeneous Turbulence (Cambridge: Cambridge University Press)

[3] Bongiovanni C 1996 Traitement d'image appliqué à la mesure de la taille, de la vitesse et de la déformation des bulles $P h D$ Thesis INP Toulouse

[4] Cohen L D 1991 On active contour models and balloons Comput. Visiont Graphical Image Process.: Inage Understanding $\mathbf{5 3} 211-18$

[5] Coulaloglou C A and Tavlarides L L 1977 Description of interaction processes in agitated liquid-liquid dispersions Chem. Eitg. Sci. 32 1289-97

[6] Friebe C A, Van Atta C. W and Gibson C H 1972 Jet turbulence: dissipation rate measurements and correlations Turbulence Shear Flows, AGARD Conf. Proc. vol 93 p 18
[7] Gibson M M 1963 Spectra of turbulence in a round jet J. Fluid Mech. 15 161-73

[8] Hinze J O 1975 Turbulence (New York: McGraw-Hill)

[9] Kolmogorov A N 1949 On the breakage of drops in a turbulent How Dokl. Akad. Nauk. SSSR 66828

[10] Konno M. Matsasunaga Y, Arai K and Saito S 1980 Simulation model for breakup process in agitated tank J. Chem. Eng. Jpu $1367-73$

[11] Lasheras J C, Eastwood C, Martínez-Bazán C and Montañés J L 2002 A review of statistical models for the break-up of an immiscible fluid immersed into a fully-developed tubulent flow Itt. I. Multiphase Flow 28 247-78

[12] Lecuona A, Sosa P A, Rodríguez P A and Zequeira R I 2000 Volumetric characterization of dispersed two-phase flows by digital image analysis Meas. Sci. Techtol. $111152-61$

[13] Luo $H$ and Svendsen H F 1996 Theoretical model for drop and bubble break-up in tutbulent dispersions $A I C h E, I .42$ $1225-33$

[14] Martínez-Bazán C 1998 Splitting and dispersion of bubbles by turbulence $P h D$ Thesis University of California, San Diego

[15] Martínez-Bazán C, Montañés J L and Lasheras J C 1999 On the breakup of an ait bubble injected into a fully developed turbulent flow. Part I: breakup frequency J. Fhid Mech. 401 $157-82$

[16] Martínez-Bazán C, Montañés J L and Lasheras J C 1999 On the breakup of an ait bubble injected into a fully developed turbulent flow. Part II: size PDF of the resulting daughter bubbles I. Fluid Mech. $401183-207$

[17] Prince M J and Blanch H W 1990 Bubble coalescence and break-up in air-sparged bubble columns $A / C h E I .36$ 1485-99

[18] Shen L, Song X. Manabu I and Yamamoto F 2000 A method for recognizing particles in overlapped particle images Pattern Recognition Lett. 21 21-30

[19] Soille P 1999 Morphological Inage Analysis: Principles and Applicationts (Berlin: Springet)

[20] Tsouris C and Tavlarides L L 1994 Breakage and coalescence models for drops in turbulent dispersions $A / C h E I .40$ $395-406$

[21] Vetnon D 1991 Machite Vision: Automated Visual Inspection and Robot Vision (New York: Prentice Hall)

[22] Williams F A 1985 Combustion Theory (Reading. MA: Addison-Wesley) 